\title{
The premetric approach to electromagnetism in the 'waves are not vectors' debate
}

\author{
Alain Bossavit \\ Laboratoire de Génie Électrique de Paris, \\ CNRS \& Univ. Paris-Sud, Orsay, France \\ *corresponding author, E-mail: bossavitelgep.supelec.fr
}

\begin{abstract}
A plea for the introduction, in advanced electromagnetics courses, of some basic differential geometric notions: covectors, differential forms, Hodge operators. The main advantages of this evolution should be felt in computational electromagnetism. It may also shed some new light on the concept of material isotropy.
\end{abstract}

\section{Introduction}

This is an expanded version of an intervention within the session "Waves are not vectors" of the AES 2012 meeting of April 18, 2012, in Paris. At a time when physicists talk about scalar or vector bosons, pseudovector mesons, and so forth, to say nothing of vector potential or Poynting "vector", it's a good idea to thus challenge this habit to confuse words and things. Not that the confusion is terribly damaging, of course. We know, don't we, what we are talking about, it's only the taxpaying common person who risks being confused - should they try to understand a bit of the science we pretend to possess and to teach. And it's inevitable to represent physical entities we wish to understand by mathematical objects, since only the latter can be handled within a logic-based formal discourse. What is at stake is the choice of these mathematical objects.

For choice there is: Several formalisms exist, within which classical electrodynamics can be described: A quaternionic one was popular before Heaviside's reformulation in use nowadays, based on vector fields. Some authors prefer tensors. Others favor Clifford algebras. Here, I will use differential forms, and try to explain why this is the best choice.

\section{Differential forms as field descriptors}

The criterion in this respect is the time-honored one of parsinomy: Introduce abstract entities only when adequate to the physical entities one wants to deal with, and only when it becomes necessary. Therefore, a well structured theory will start with weak mathematical structures and enrich them on demand, as the theory grows, as if painting layer over layer of the desired theoretical framework. Rather than unwrapping this metaphor, let's have an example.

Consider the case of the electric field $E(x)$ at a point $x$ where it is well defined (i.e., not at a material interface). This vector tells about the force exerted by the field over a unit electric charge placed at $x$. This force itself is known, to first order, via the virtual work $E(x) \cdot v$ involved in moving the charge from $x$ to $x+v$, where vector $v$ stands for the virtual displacement. The mapping $v \rightarrow v \cdot E(x)$ being linear and continuous on the space $V$ of three-dimensional vectors, it is - by the very definition of a dual - some element $e(x)$ of the dual $V^{*}$ of $V$. Such elements are called covectors and the pairing between a vector $v$ and a covector $\omega$ is denoted by $\langle v ; \omega\rangle$. So we have here $v \cdot E(x)=$ $\langle v ; e(x)\rangle$, showing that the electric field, as a physical entity, is totally described by the field (in the mathematical sense) $x \rightarrow e(x)$, a field of covectors. (We denote this field by $e$ in what follows. It's called a differential form of degree one, or 1-form. Cf., e.g., [1, 2].) Comparing now the two sides of the equality $v \cdot E(x)=\langle v ; e(x)\rangle$, we see two possible mathematical representations of the electric field: $\left(1^{\circ}\right)$ The one on the left, which uses the vector field $E$ in association with the metric structure conferred on space by the dot product ".", $\left(2^{\circ}\right)$ The one on the right, which uses the 1 -form $e$ plus nothing else. Parsimony mandates $\left(2^{\circ}\right)$ as the best choice: $E$ appears as an auxiliary entity, that plays the role of proxy for the real thing, $e$.

To show how the 1-form $e$ (taken at some instant of time, of course) gives complete information on the electric field, let's work out the electromotive force (e.m.f.) along some oriented curve $c$. For this, chop $c$ into a sequence of segments $\left\{x_{i-1}, x_{i}\right\}$, with $x_{0}$ at the start-point of $c$ and $x_{n}$ at the end-point, call $v_{i}$ the vector from $x_{i-1}$ to $x_{i}$, and form the sum $\sum_{i}\left\langle v_{i} ; e\left(x_{i-1}\right)\right\rangle$. As the subdivision of $c$ is properly refined, with $n$ going to infinity, this Riemann sum tends to a number, that we shall denote either by $\int_{c} e$ (the integral of $e$ along $c$ ), or $\langle c ; e\rangle$, to emphasize the duality pairing between the integration domain $c$ and the integrandum $e$. This limit number is the e.m.f. along $c$ : It's what a voltmeter, whose connecting threads would follow the path $c$, would display. Since $c$ can be any (smooth enough) oriented curve, the 1-form $e$ does indeed know all that is physically meaningful about the electric field.

Note that $\langle c ; e\rangle$ is also the number that would be obtained by evaluating the so-called circulation of the proxy field $E$, that is to say, the integral $\int_{c} \tau(x) \cdot E(x) \mathrm{d} x$, where $\tau(x)$ is the tangent vector of length 1 at point $x$ of curve $c$, oriented in the forward direction along $c$, and $\mathrm{d} x$ the mea- 
sure of lengths. Comparing these two expressions of the e.m.f., one sees that the metric of space provided by the dot-product ". " is not essential to the physical description of the field: One could change this metric for a different one, with dot product " .' ", say, and then, one would have to describe the field by a different proxy vector-field $E^{\prime}$, and to equip the curve $c$ with a different set $\tau^{\prime}$ of tangent vectors and a different lineal measure $\mathrm{d}^{\prime} x$, in order to preserve the equality $\int_{c} \tau(x) \cdot E(x) \mathrm{d} x=\int_{c} \tau^{\prime}(x) \cdot E^{\prime}(x) \mathrm{d}^{\prime} x$ between these two different expressions for the same e.m.f. $\langle c ; e\rangle$. Fields are not vectors, therefore: A vector field like $E$ is not the mandatory representation of the electric field, as a physical entity. The 1-form $e$ does the job much better.

Similar considerations apply to the magnetic induction. One fully knows it when, for any oriented surface $S$, the induction flux $\langle S ; b\rangle$ embraced by $S$ is known. Faraday's law then connects the rate of variation of this flux with the e.m.f $\langle\partial S ; e\rangle$ along the boundary $\partial S$ of $S$ :

$$
\mathrm{d}_{t}\langle S ; b\rangle+\langle\partial S ; e\rangle=0
$$

(Beware that $S$ must not change in time for this to hold, so that one has $\mathrm{d}_{t}\langle S ; b\rangle=\left\langle S ; \partial_{t} b\right\rangle$.) Orienting the surface means providing it with a gyratory sense (clockwise or counterclockwise), and orientations of $S$ and its boundary should "match", that is, the forward direction along $\partial S$ should respect the gyratory sense assigned to $S$.

[Some technical details, which the reader can skip, would be required to parse this compact expression $\langle S ; b\rangle$. First, a bivector (or 2-vector) $u \vee v$ (the "join" of vectors $u$ and $v$, both anchored at the same point of space) is the algebraic object represented by the parallelogram spanned by $u$ and $v$, with orientation (i.e., gyratory sense) provided by the rule "go along $u$ first, then turn to direction $v$ ". Bivectors at a point form a vector space, whose dual elements are called bicovectors, or 2-covectors. Starting from two covectors $\omega$ and $\eta$, one may form a 2-covector $\omega \wedge \eta$ by the rule $\langle u \vee v ; \omega \wedge \eta\rangle=\langle u ; \omega\rangle\langle v ; \eta\rangle-\langle v ; \omega\rangle\langle u ; \eta\rangle$. A differential form of order 2 , or 2 -form, is a smooth field of 2-covectors. To understand $\langle S ; b\rangle$, where $b$ is such a 2form, imagine surface $S$ chopped into small patches $u_{i} \vee v_{i}$, each based at point $x_{i}$ of $S$, and form the Riemann sum $\sum_{i} \pm\left\langle u_{i} \vee v_{i} ; b\left(x_{i}\right)\right\rangle$, where the sign in front of $u_{i} \vee v_{i}$ is + if this patch has the same orientation as $S$ itself, - otherwise. The limit of that is, by definition, $\langle S ; b\rangle$.]

Although such cumbersome details are needed in the theory, one can easily bypass them: Just think of $e$ and $b$ as mappings from oriented curves or surfaces to reals, the value being interpreted as an e.m.f. or a flux. These maps are linear (and also continuous, in some rather involved sense). Linearity means that, for instance, $\left\langle c_{1} ; e\right\rangle+\left\langle c_{2} ; e\right\rangle$ $=\left\langle c_{1}+c_{2} ; e\right\rangle$, where $c$ is the curve obtained by chaining $c_{1}$ and $c_{2}$ : This linearity reflects the fact that, in case the end-point of $c_{1}$ is the start-point of $c_{2}$, a voltmeter would measure for this chain the sum of the e.m.f.'s measured along $c_{1}$ and $c_{2}$ separately. Hence the concept of chain: a weighted sum, with real coefficients, of oriented submanifolds of common dimension $p(p=1$, in our example, with both coefficients equal to +1 ) and of cochain: a $p$-cochain is an element of the dual of the space of $p$-chains. Examples are the 1-cochain $e$ and the 2-cochain $b$. Zero-cochains are just functions (such as the scalar electric potential $\psi$ ). The expressions " $p$-form" and " $p$-cochain" can be taken as synonyms.

Thanks to the linear structure thus conferred to chains, one may consider the boundary operator $\partial$ as a linear map, from $p$-chains to $(p-1)$-chains, which can be useful: For instance, the boundary of curve $c$ joining point $p$ to point $q$, considered as a 1-chain, is the 0 -chain $\partial c=q-p$. If surface $S$ is a cylinder bounded by two closed curves $c_{1}$ and $c_{2}$, then $\partial S$ is a chain based on $c_{1}$ and $c_{2}$, like e.g. $c_{2}-c_{1}$, depending on how $S, c_{1}$ and $c_{2}$ are oriented. (The orientation of $\partial S$, which must match that of $S$, is thus determined, but it may not match with the orientations of the various components of the chain $\partial S$. Hence the possible minus signs.) Also, in finite element practice, one will often deal with surfaces made of triangular facets of the mesh. As mesh elements are independently oriented, such surfaces will be 2 -chains based on mesh facets, with weights \pm 1 (or 0 , for facets lying outside the surface), and their boundaries will be edge-based 1-chains, also with weights \pm 1 or 0 .

\section{Exterior derivative, Maxwell's equations}

But the main advantage of this linearity is perceived in what follows: Define a new operator denoted d, called exterior derivative, from $(p-1)$-cochains to $p$-cochains, by the clause

$$
\langle S ; \mathrm{d} e\rangle=\langle\partial S ; e\rangle \text { for all } p \text {-chains } S,
$$

that is to say, define $\mathrm{d}$ as the dual of $\partial$. (This equality is the general form of the Stokes theorem.) Turning to (1), where $S$ was but a dummy variable, we can get rid of it, and transform (1), using d, into the following local expression of Faraday's law:

$$
\partial_{t} b+\mathrm{d} e=0
$$

which is as simple as possible. In particular, no metric element is used in it, contrary to what happens in the standard expression $\partial_{t} B+\operatorname{rot} E=0$.

There, $B$ plays proxy in a way similar to what we already described about $E$. The flux $\langle S ; b\rangle$ embraced by $S$ can be written as $\int_{S} n(x) \cdot B(x) \mathrm{d} x$, where $\mathrm{d} x$ is the areal measure and $n$ one of the two fields of unit-length vectors normal to $S$. This normal field has to be chosen in such a way that (applying the right-hand rule), the gyratory sense indicated by counter-clockwise rotation around $n$ coincides with the orientation of $S$. Then, the equality $\int_{S} n \cdot \operatorname{rot} E=$ $\int_{\partial S} \tau \cdot E$ comes from the elementary version of the Stokes theorem, and (1) is seen to result in $\partial_{t} B+\operatorname{rot} E=0$. All the objects we are dealing with here, $E, B, \tau, n$, the curl operator rot, are metric-dependent, but these dependencies appear to cancel each other, resulting in the completely metric-free expression (3)!

The behavior of $B$, as a proxy field for $b$, is more complex than that of $E$ : Not only $B$ will have to change if the 
metric is changed, in order to keep the flux $\langle S ; b\rangle$ invariant, but with unchanged metric the sign of $B$ will still depend on which orientation convention (right-hand or left-hand) is adopted. Orientation of the ambient three-dimensional space (that is, the choice of which species of screws, snails, stairs, etc., are deemed right-handed or left-handed) is a convention which has no necessary connection with the choice of metric and/or Cartesian axes, but quite often-by choice of the analyst, not by physical necessity-all these elements go together: The basis of vectors $w_{1}, w_{2}, w_{3}$ one selects is supposed to determine the scalar product (by the rule $u \cdot v=\sum_{i} u^{i} v^{i}$, if $u=\sum_{i} u^{i} w_{i}$ and $\left.v=\sum_{i} v^{i} w_{i}\right)$, and is supposed to be right-handed. Changes of basis-which cannot have any physical import - then wreak havoc in the relations between mathematical entities, hence these arcane notions of "axial" (vs "polar") vectors, of right-handed vs left-handed cross products, etc., that make the teaching of Electromagnetism so taxing. As (1) demonstrates, all these props can be discarded.

By analogy, one can guess that Ampère's law, $-\partial_{t} D+$ $\operatorname{rot} H=J$ in standard terms, must be replaced by

$$
-\partial_{t} d+\mathrm{d} h=j,
$$

where $h$ is a 1 -form and $d$ and $j$ are 2 -forms. There is however an important difference. For $j$ to properly represent current density, its integral $\int_{\Sigma} j$, or $\langle\Sigma ; j\rangle$ in the alternate notation, over a surface $\Sigma$, should represent an intensity going through $\Sigma$, which implies a crossing direction with respect to $\Sigma$, not the same thing at all as the gyratory sense that was necessary on $S$ for $\langle S ; b\rangle$ to make sense. Of course, if the ambient space is oriented, the two kinds of orientation are related by the right-hand rule, but why should one impose this conventional, human created, feature-an orientation of the space we inhabit-in background of a physical theory, if it can be avoided?

It can, as follows: Let's distinguish outer orientation, characterized in the case of surfaces by a crossing direction, from inner orientation, characterized by a gyratory sense. Two-chains based on outer-oriented surfaces will be called twisted 2-chains [3], those based on inner-oriented ones being known from now on as straight 2-chains, for due contrast. A similar distinction holds for 1-chains: An outer orientation, for a curve $\gamma$, is a way to turn around it (instead of going along it) in a definite sense, that is to say, a consistent system of inner orientations of closed loops encircling $\gamma$. Twisted 1-chains are those based on such outer-oriented curves. Now if $\gamma$ is the boundary $\partial \Sigma$, the outer orientations of $\Sigma$ and $\partial \Sigma$ "match" when the forward direction along such an encircling loop coincides with the crossing direction assigned to $\Sigma$. Finally, twisted cochains are defined as mappings from twisted chains to reals, the $\mathrm{d}$ being again the dual of $\partial$, and eq. (4) can be interpreted as a relation between the $\sim 1$-form $h$ (note the use of $\sim$ as a shorthand for "twisted") and the $\sim 2$-forms $d$ and $j$.

The real number $\langle\gamma ; h\rangle$ is the magnetomotive force (m.m.f.), not "along" $\gamma$, like an e.m.f., but "around" it, so to speak, which makes sense when one thinks that an m.m.f. is created by Ampère turns, i.e., current flows, that do "turn around" $\gamma$ in a clearly defined way. Symmetrically, the induction flux $\langle S ; b\rangle$ is not "through" $S$, contrary to what its standard representation $\int_{S} n \cdot B$ tends to suggest, with this $n$ that looks like a crossing direction. The meaningful direction is instead the forward one along the boundary $\partial S$, which abstractly represents the way one arranges along $\partial S$ the threads of the voltmeter that records-thanks to Faraday's law - the rate of change of the magnetic flux. Hence the word "embraced" to correct, as much as possible, the wrong suggestion that magnetic flux would be, like intensity, the flux of some kind of "stuff" across a surface. This interpretation, on the other hand, is all right as $j$ is concerned, for $\langle\Sigma ; j\rangle$ is the quantity of electric charge that crosses $\Sigma$, per unit of time. Electric charge $q$, by the way, is a twisted 3-form, the integral $\langle\Omega ; q\rangle$ of which over a volume $\Omega$ represents the total charge inside $\Omega$. A straightforward application of the Stokes theorem shows then that

$$
\partial_{t} q+\mathrm{d} j=0
$$

expresses conservation of electric charge. Charge density $q$, here, rather than being a new entity in the theory, is defined from $d$ by $q=\mathrm{d} d$, so one can see, by applying $\mathrm{d}$ to both sides of (4), that (5) derives from (4), thanks to the relation $\mathrm{d} \circ \mathrm{d}=0$, which itself is a consequence, by duality, of the obvious property $\partial \circ \partial=0$, "the boundary of a boundary is zero" [4].

Magnetic charge, if such a thing existed, would similarly be represented by the (straight, this time) 3 -form $m=\mathrm{d} b$. The fact that $\mathrm{d} b=0$ is, again, seen to be a consequence of Faraday's law by letting $d$ act on both sides of (3), under the natural assumption that $b$ has been null until some instant $t$, for instance $t=0$. We have all reasons to believe that $m=0$ indeed [5], but if experience said otherwise some day, adapting Maxwell's theory to that would be easy: Just put, on the right-hand side of (3), a term $-k$, where $k$ is a (straight) 2-form, interpreted as the current of magnetic charge [6]. Then, magnetic charge would be conserved, $\partial_{t} m+\mathrm{d} k=0$, as a consequence of (4).

A last remark on the straight-vs-twisted opposition: straight and twisted forms appear in other fields of physics. In Thermodynamics, for instance, temperature is a 0 -form, its gradient is (the proxy of) a 1-form, heat flux is a twisted 2 -form, and volumic enthalpy is a $\sim 3$-form. Entropy has the same status. One can observe on this that so-called "intensive variables" always correspond to straight forms, while "extensive" ones are twisted forms. This is no accident, and straight [resp. twisted] $p$-forms are proper generalizations of the concept of intensive [resp. extensive] quantities.

\section{Constitutive laws}

The metric structure thus appears as superfluous, so far. Worse, using it when stating Maxwell's equations hides some important things, such as the difference in nature just evoked between intensive and extensive entities. Yet, the $\square$ 
metric thus expelled comes back, in an interesting way, in the constitutive laws.

We expect, of course, two relations such as $h=\nu b$ and $d=\epsilon e$ to complete (3) and (4). But $\nu$ and $\epsilon$ there can no longer be mere numbers, since they connect objects of different types. They must be, accordingly, operators: What we have, at each point $x$ of space, is a map $\nu(x)$ from straight 2-covectors to twisted covectors, and a map $\epsilon(x)$ from straight covectors to twisted 2-covectors. Such maps, from a $p$-covector, straight or twisted, to an $(n-p)$-covector of the opposite kind, i.e., twisted or straight, are called, when they are linear, "Hodge operators". In the use we made of them here (assuming linearity of the constitutive laws), they describe the behavior of matter (or of vacuum) at point $x$. (Ohm's law, similarly, would be $j=\sigma e+j^{s}$, where $j^{s}$ is the "source" current, the one that generates the field. But let's ignore this issue and consider $j$ in (4) as given.) They can be considered locally, at each point $x$, or globally, as in $h=\nu b$ and $d=\epsilon e$, thus sending a $p$-cochain to an $(n-p)$-cochain.

As it appears (we soon return to this point), $\epsilon$ and $\nu$ encode the metric structure of space-time (they determine the light cone, in particular). So at this stage one has a neat separation between the so-called pre-metric part of Maxwell's theory (essentially (3) and (4), both conservation laws of sorts, plus derived relations such as (5) and $\mathrm{d} b=0$ ), and its metric aspects - constitutive laws - that characterize the medium in which the electromagnetic field develops, and thus appear as less fundamental than the two main equations.

Such a separation is more difficult in the classical theory: In $B=\mu H$, for instance, we have most often a scalar $\mu$, but also, sometimes, a symmetric tensor $\mu_{j}^{i}$, so that components of $B$ are given by $B^{i}=\sum_{j} \mu_{j}^{i} H^{j}$. A mere change of basis being enough to pass from a scalar to such a tensor, this casts in doubt the notion of isotropy: Isotropy cannot naively be understood as the presence of off-diagonal components in matrix $\mu$, since that could be achieved by a change of basis, an operation which is, physically, irrelevant. A more refined notion of anisotropy comes from restricting changes of basis to orthonormal ones, so that eigenvalues of $\mu$ acquire an invariant character. It then seems to make sense to identify isotropy with the equality of these three principal values. But this amounts to endow the chosen metric (in which the proxies of $b$ and $h$ are $B$ and $H$ ) with a physical character that it may not possess. The usual Euclidean metric has such a character as far as the vacuum is concerned, because our geodesy is based on the very existence of light rays, so that the metric we favor is adapted to Maxwell's equations in the void (hence the scalar character of $\epsilon_{0}$ and $\mu_{0}$ in the laws $D=\epsilon_{0} E$ and $\left.B=\mu_{0} H\right)$. But this is not necessarily the case in crystals, be it natural ones or artificial ones such as metamaterials.

\section{Anisotropy}

Hodge operators help understand this question better, to the price of conceiving them in a different way than what clas- sical textbooks propose $[1,2]$. These define a scalar product first, and then use it to build a map from $p$-covectors to $(n-p)$-covectors. Here, we do the opposite: We take as primitive objects the operators $\epsilon$ and $\mu$, as given by physics, and determine a metric adapted to each of them. Isotropy, or lack of it, is then a matter of how these (a priori different) metrics relate. Let's describe this mechanism.

So let be given (by adequate measurements) the operators $\epsilon(x)$ and $\nu(x)$ that link, at each point $x$, the local values of the fields $d$ and $h$, to those of $e$ and $b$. Owing to their relation to electric and magnetic energy density, these operators are, as a rule, symmetric and positive definite, which means that the $\sim 3$-forms $e(x) \wedge \epsilon e(x)$ and $b(x) \wedge \nu b(x)$ don't vanish for $e(x) \neq 0$ and $b(x) \neq 0$, and that (let's drop the $x$, understood, from now on) $e \wedge \epsilon e^{\prime}=e^{\prime} \wedge \epsilon e$ and $b \wedge \nu b^{\prime}=$ $b^{\prime} \wedge \nu b$ whatever $e, e^{\prime}, b, b^{\prime}$. By setting $|b|_{\nu}=\lambda(b(x) \wedge \nu b)^{1 / 2}$, where $\lambda$ is a suitable constant, one obtains a norm on the linear space of 2-covectors, from which a Euclidean norm ||$_{\nu}$ on vectors is easily derived. (Adjusting $\lambda$ to make the notions of length and of volume consistent, cf. [7], yields a unique, canonical such norm.) Hence a metric, " $\nu$-adapted" in the sense that, if $B$ and $H$ are the proxies of $b$ and $h$ for this metric, then $B=H$. "Hodge implies metric", so to speak. (This works in spatial dimensions 3 and higher, cf. [7].)

Now let's turn towards $\epsilon$. By the same process, one can form an $\epsilon$-adapted metric ||$_{\epsilon}$, which quite often will be the same as ||$_{\nu}$, up to a multiplicative constant. This is what happens for the vacuum, for which this constant is to do with its impedance. It also happens for most kinds of transparent matter, where the constant is affected by another multiplicative factor, called the refractive index of the material. In both cases, there is isotropy in a physically meaningful sense, since light rays, as it can be proven, propagate at the same velocity in all directions and whatever the polarization, even though a choice of metric non-adapted to either $\epsilon$ or $\nu$ could mask this fact.

So what about genuine anisotropy? This holds when, having chosen one of the hodges (usually, $\nu$ ) to make a metric with, the eigenvalues of the matrix $\epsilon$ that links the proxies $D$ and $E$ of $d$ and $e$ by $D=\epsilon E$, happen to be distinct. (Proxies $D$ and $E$ there, of course, are those relative to the $\nu$-metric.) Then the velocity of light (as measured by using the $\nu$-metric) depends on direction, and the very notion of light cone can even collapse if the three principal values differ two by two, to be replaced by a more complex geometrical object, the Fresnel surface [6]. Propagation velocity of a plane wave then also depends on its polarization, hence the spectacular phenomenon of birefringence [8].

Isometry, to sum up, is not a matter of commutativity between $\epsilon$ and $\nu$, or its inverse $\mu$ (the products $\epsilon \nu$ or $\nu \epsilon$ do not even make sense). It's a matter of both determining the same metric, up to a multiplicative factor, of 3D space. This amounts to saying that $\epsilon$ and $\mu$ are the same up to such a factor, a criterion which can be applied whatever the representation of these operators. So we may compare, for instance, the standard representations of $\epsilon$ and $\mu$ as Euclidean 
tensors, $\epsilon_{j}^{i}$ and $\mu_{j}^{i}$, in a system of orthonormal coordinates: Isotropy holds if and only if there is a constant $r$ such that $\epsilon_{j}^{i}=r \mu_{j}^{i}$. This does not mean that the matrices $\epsilon_{j}^{i}$ and $\mu_{j}^{i}$ are scalar multiples of the identity.

So one may conceive of metamaterials whose effective permittivity and permeability would not be isotropic in the common sense, but still would propagate waves the way an isotropic medium does, that is, with spherical wavefronts expanding away from each emitter point. But 'spherical' here refers to the intrinsic metric of the metamaterial, the one determined, up to a constant, by the Hodge operators $\epsilon$ and $\mu$, and these wavefronts would not appear to us outsiders as spheres, but as ellipsoids.

\section{Conclusion and prospects}

This geometric view of the Maxwell equations we have sketched here is almost a century old $[3,9,10,11]$ and has not been adopted yet in mainstream treatises. It should be. It's the best way to present modern numerical techniques, be they based on the Galerkin method with edge elements, on finite volume ideas, or on so-called "mimetic" heuristics [13]. The above viewpoint leads naturally to such methods, which can be presented under the common header "Generalized Finite Differences" [12]. The salient points are $\left(1^{\circ}\right)$ By attaching degrees of freedom to elements of the mesh (not only and exclusively nodes, but also edges, facets, volumes), one is led, almost compulsorily, to a discrete expression of the conservation laws (3) and (4), which in a sense introduces no approximation error, $\left(2^{\circ}\right)$ The Hodge operators $\epsilon$ and $\nu$ must be approximated by square matrices (indexed over edges and facets, respectively), and it's in this process that approximation errors appear. So the central task of the theory, from the numerical point of view, consists in approximating Hodge operators in a consistent way, and this is where different techniques may compete. This is also where geometric objects introduced for purely mathematical purposes long ago, such as Whitney forms [14], become useful.

Some benefits can be drawn from this also when dealing with waves, and in metamaterial studies. A plane wave is a time-varying field of covectors of the form $e(t, x)=$ $\operatorname{Re}[\operatorname{Eexp}(i(\omega t-\langle x ; \kappa\rangle))]$, where both $\mathrm{E}$ and $\kappa$ are covectors, and a similar expression for $h$. Again, no metric element there, contrary to what happens in the standard formalism where $x \cdot \kappa$, with $\kappa$ a vector, would replace $\langle x ; \kappa\rangle$. Treating $\kappa$ as a covector shifts emphasis from the direction of propagation of the wave (which is along the vector $\kappa$ in the standard treatment) to the planes of equal phase (those parallel to the kernel of the covector $\kappa$ ). This small difference eases up the treatment of wave propagation in anisotropic media, including the study of dispersion relations. In particular, a streamlined treatment of the Fresnel surface is obtained. It's even more true with Bloch waves when studying crystal-like media (replace $\mathrm{E}$ above by a cell-periodic covector field $\mathrm{E}(x)$ borne by a Bravais cell). The difference between Bravais cells (parts of $V$ ) and Brillouin zones (parts of $V^{*}$ ) is better marked and metric properties are not in the way.

Fields, to sum up, are physical entities, and there exist mathematical entities with the same name that can represent them. The latter are our creation, and should be chosen with care. Differential forms, aka cochains, i.e., fields of covectors ( $b i$-covectors in some cases, as has been explained) are the recommended choice. Other mathematical entities, namely fields of special form called "waves" (plane waves, spherical waves, Bloch waves ...) also are useful (when taken as wave packets) to represent physical fields, but just that. Fields are not waves, waves are not vectors, not even vector fields, vector fields "are" not the physical fields they stand proxy for. One should not reify vector fields (or differential forms, for that matter) and believe in their existence as physical objects.

\section{References}

[1] W.L. Burke, Applied Differential Geometry, Cambridge University Press, Cambridge (U.K.), 1985.

[2] Th. Frankel, The Geometry of Physics, An Introduction, Cambridge U.P., Cambridge (U.K.), 1997.

[3] W.L. Burke, Manifestly parity invariant electromagnetic theory and twisted tensors, J. Math. Phys., 24: 65-69, 1983.

[4] C.W. Misner, K.S. Thorne, and J.W. Wheeler, Gravitation, Freeman, New York, 1973.

[5] A.S. Goldhaber and W.P. Trower, Resource Letter MM-1: Magnetic monopoles, Am. J. Phys., 58: 429439, 1990).

[6] F.W. Hehl and Y.N. Obukhov, Electric/magnetic reciprocity in premetric electrodynamics with and without magnetic charge, and the complex electromagnetic field, Phys. Lett. A, 323: 169-175, 2004.

[7] A. Bossavit, On the notion of anisotropy in constitutive laws: some implications of the 'Hodge implies metric' result, COMPEL, 20: 233-239, 2001.

[8] A. Fresnel, Mémoire sur la double réfraction, Mémoires de l'Acad. de l'Institut de France, 7: 45$176,1827$.

[9] F. Kottler, 'Maxwell'sche Gleichungen und Metrik, Sitzungber. Akad. Wien IIa, 131: 119-146, 1922.

[10] E.J. Post, "Kottler-Cartan-van Dantzig (KCD) and Noninertial Systems", Found. Phys., 9: 619-640, 1979.

[11] M.J.A. Schouten and D. Van Dantzig, On ordinary quantities and $W$-quantities, Compositio Math., 7: 447-473, 1939.

[12] A. Bossavit, Discretization of Electromagnetic Problems: The 'Generalized Finite Differences Approach', 
in W.H.A. Schilders, E.J.W. Ter Maten (Eds): Numerical Methods in Electromagnetism (Handbook of Numerical Analysis, Vol. 13), Elsevier, Amsterdam, pp. 105-197, 2005.

[13] J.H. Hyman and M. Shashkov, Mimetic Discretizations for Maxwell's Equations, J. Comp. Phys., 151: 881-909, 1999.

[14] H. Whitney, Geometric Integration Theory, Princeton U.P., Princeton, 1957. 